\title{
Les modes de gestion possibles du transport en commun
}

\author{
Jacques Gagnon et Peggy Bachman ${ }^{1}$ \\ Université deSherbrooke
}

La problématique du transport en commun pose plusieurs défis intéressants aux gestionnaires publics et aux experts. Ces défis sont souvent très différents selon que l'on se place dans la problématique des pays occidentaux ou dans celle des pays d'Amérique latine.

En effet, dans plusieurs pays occidentaux, comme le Canada et les États-Unis, la gestion du transport en commun implique, dans la majorité des grandes et des moyennes villes, l'élaboration de politiques publiques visant à planifier des services de transport déjà regroupés dans des institutions publiques ou parapubliques. Or, dans la plupart des pays d'Amérique latine, les enjeux sont plutôt de regrouper une multitude de petits opérateurs privés pour parvenir à une meilleure coordination du service.
Plusieurs approches financières et de gestion ont été proposées à ce jour pour tenter d'améliorer la situation, dans le but de maintenir un développement durable. Peu d'hypothèses ont comporté comme élément de solution l'approche de la gestion coopérative dans le domaine du transport en commun. Pour cette raison, d'un point de vue scientifique, nous avons cru intéressant d'introduire cette approche dans l'analyse de la problématique du transport en commun en Amérique latine, ainsi que dans la recherche de solutions à moyen et à long termes.

Notre objectif est de rechercher des solutions de gestion applicables au transport en commun à l'intérieur d'une approche générale de gestion du développement. Cette approche est traitée sous l'angle du retour vers la territorialité, que nous présenterons dans un

Dans les pays occidentaux, dans le domaine de la gestion des ressources financières, on parle davantage de problématiques liées au partage des recettes fiscales à l'échelle régionale entre les différents gestionnaires locaux (ex.: tax base sharing, tax free bonds, taxe régionale, système de redevances). Dans les pays du sud, la problématique est différente, puisque l'on tente d'imaginer des structures financières ou des montages financiers qui pourraient permettre la viabilité à court et à moyen termes d'organismes de gestion du transport en commun, capables d'assurer un meilleur service aux usagers.

Dans le contexte plus difficile des pays en développement, les solutions sont souvent ardues à identifier, faute de ressources financières et techniques. Cependant, il est vital pour ces pays d'y parvenir, car le transport en commun demeure l'un des facteurs majeurs de développement économique et social. premier temps. Elle suggère une gestion particulière du développement local qui, nous croyons, convient parfaitement aux principes promus dans le milieu coopératif. Ainsi nous expliquerons dans un deuxième temps en quoi le mode de gestion coopératif devrait être privilégié dans la gestion du transport en commun, plus particulièrement en Amérique latine. Enfin nous aborderons des pistes de solutions complémentaires à la gestion coopérative, et envisageables pour la gestion du transport en commun dans un contexte de retour vers la territorialité.

\section{Le contexte de la gestion des services publics: le retour vers la territorialité}

Avant d'envisager les choix potentiels dans les méthodes de gestion du transport en commun, il est important, dans un premier temps, de déterminer les principaux facteurs de modernisation de l'administration publique afin de 
mieux se situer dans le contexte global du changement. En effet, la gestion du transport en commun relève de la problématique de la gestion des services publics, le transport en commun constituant en lui-même un service public, qu'il soit géré par des entités privées ou par des entités publiques. Ainsi, le contexte global de la modernisation de l'administration publique nous permettra de mieux évaluer les solutions potentielles à la problématique liée au transport en commun.

Par ailleurs, les stratégies de développement dans tous les pays sont de plus en plus élaborées à l'échelle locale. Auparavant, les politiques publiques étaient principalement l'émanation des gouvernements centraux, mais ceux-ci ont échoué dans plusieurs domaines d'activité, et ils ont dû céder des pouvoirs qui étaient jadis dévolus à d'autres niveaux institutionnels (collectivités locales, coopératives, entreprises privées, etc.).

Pourquoi le local devient-il si important dans l'élaboration des stratégies de développement économique, social et institutionnel ? La réponse à cette question réside, de notre point de vue, dans le fait que le local constitue la pierre angulaire d'un processus de changement, qu'il est possible de désigner par la formule «retour vers la territorialité».

Le local constitue la pierre angulaire d'un processus de changement, qu'il est possible de désigner par la formule « retour vers la territorialité».

\section{Le concept de « retour vers la territorialité»}

Le retour vers la territorialité se veut une manière de solutionner plusieurs défis de la gestion globale du développement. Le territoire devient un élément clé du développement social et économique. Les décisions doivent être prises en grande partie à partir du milieu, et les stratégies de développement, même à l'échelle internationale, devraient en être issues dans une grande majorité.

$$
\begin{aligned}
& \text { On pourrait définir le retour vers la } \\
& \text { territorialité comme un mouvement de } \\
& \text { décentralisation et ou de déconcentration de } \\
& \text { I'administration publique pour se } \\
& \text { repositionner par rapport au territoire. }
\end{aligned}
$$

Le retour vers la territorialité comprend cinq facteurs principaux de changement ou de modernisation de l'administration publique:

\section{1) L'échec des politiques publiques}

La question du territoire est au cœur des débats économiques et sociaux. Le management public vit depuis plusieurs années une crise. Les fondements mêmes des grandes théories contemporaines de management sont mis en doute, et la confiance, parfois illimitée, a fait place à la méfiance et à la suspicion.

Les politiques sectorielles, après avoir souvent fait preuve d'inefficacité, font graduellement place au concept de la territorialité, un mouvement qui fait en sorte qu'on délaisse les politiques publiques pour revenir au concept de territoire dans la gestion administrative publique. Ce retour de la territorialité peut nous paraître rétrograde sous certains aspects, mais dans le contexte des grands marchés internationaux et d'une société d'information sans cesse croissante, il est clair que ce retour devient la voie à suivre en remplacement du management traditionnel. Ce mouvement de la territorialité inclut donc le constat d'échec du management public ${ }^{2}$ et la nécessité de trouver une autre voie qui vise à mettre de côté les grandes politiques publiques et à se baser plutôt sur des administrations publiques déconcentrées ou décentralisées plus proches du milieu pour en arriver à une meilleure gestion du territoire. L'administration du territoire doit aussi reposer sur des structures fortes pour occuper ces nouveaux champs de compétence, et les régions et les collectivités locales constituent selon nous les entités toutes désignées dans le cadre d'une administration déconcentrée ou décentralisée.

Comme nous l'avons vu antérieurement, les régions et les collectivités locales nord-américaines doivent prendre en main plusieurs secteurs où a été constaté l'échec des grandes politiques publiques, notamment la formation de la main-d'œuvre, la sécurité, l'habitation, l'intégration des nouveaux immigrants, la construction de grandes infrastructures publiques afin d'être capables d'améliorer leur potentiel de développement et celui du pays tout entier. Pour y arriver, il s'avère nécessaire d'améliorer la situation financière de ces nouveaux intervenants privilégiés que sont les régions et les métropoles. 


\section{2) La mondialisation des marchés}

Ce retour vers la territorialité de l'administration est influencé par un facteur important de changement : la mondialisation des marchés. En effet, les frontières des pays revêtent de moins en moins d'importance et les échanges s'effectuent de villes à villes ou de régions à régions. L'administration rencontre de plus en plus de difficulté à mettre de l'avant de grandes politiques d'ensemble. La territorialité dans une administration déconcentrée ou décentralisée plus efficace et plus apte à répondre aux défis d'adaptation posés à nos sociétés pour faire face à la compétition internationale devient le nouveau mode de gestion privilégié dans le secteur public.

C'est pour cette raison qu'il faudrait au moins en partie délaisser les grandes politiques publiques. Dans ce cas encore, les régions et les métropoles se présentent comme les interlocuteurs à privilégier.

\section{3) L'augmentation des moyens de communication}

Aujourd'hui les distances sont de moindre importance. Grâce à la technologie, les individus peuvent travailler à partir de leur résidence et communiquer dans le monde entier. Les concepts du milieu de travail et des centres d'affaires sont en plein changement. La région autrefois éloignée devrait désormais, par la technologie de l'information, être en mesure de se développer et de concurrencer, dans certains domaines, des centres urbains importants.

Grâce à ce changement, l'individu est pour la première fois depuis longtemps en mesure, comme avant, de travailler dans son milieu. Le territoire regagne une importance et une autonomie qu'il avait perdues au profit des grands centres de décisions.

On peut à nouveau penser à un développement qui trouverait son origine au niveau du territoire. La mondialisation des marchés, alliée au développement de la technologie, consacre à nouveau la nécessité d'établir une stratégie de développement axée sur le territoire et sur les régions. C'est un retour vers la territorialité!

\section{4) L'endettement des gouvernements supérieurs}

Les gouvernements supérieurs ne possèdent plus les ressources nécessaires à l'établissement de systè- mes de péréquation adéquats. L'endettement de ces instances supérieures a forcé un désengagement de ceux-ci dans la gestion des affaires publiques. Il a fallu faire autant avec moins de ressources. De nouveau, un retour vers le territoire s'avère une solution pour prendre en main ces secteurs et réaliser une gestion plus efficace répondant vraiment aux besoins du milieu.

\section{5) L'étalement urbain et la décadence} des grandes métropoles

En plus des grands facteurs de changements déjà évoqués, il en existe un autre plus spécifique aux sociétés nord-américaines et qui s'étend à certaines parties de l'Europe et aussi à certains pays en développement. Ce mouvement impose de se tourner vers la territorialité et de délaisser les grandes politiques publiques. Il correspond au problème de l'étalement urbain, qui accule certaines grandes métropoles à la faillite financière et qui les amène à la limite du point de rupture.

Le retour à la territorialité passe par un redécoupage de l'assiette fiscale à l'échelle régionale pour obtenir un meilleur équilibre financier, et aussi pour obtenir les instruments permettant de contrer l'étalement urbain. Il devrait idéalement être appuyé par une déconcentration financière du gouvernement central vers la région urbaine, afin de mieux gérer ce territoire. Il devrait également s'accompagner de la mise de côté de certains principes du fédéralisme fiscal ${ }^{3}$.

6) Les conséquences du retour vers la territorialité sur la gestion publique

Pour compléter la définition générale du retour vers la territorialité, soulignons que la territorialité est un concept de science administrative. En effet, on peut dire que coordonner, c'est territorialiser'. C'est aussi un concept social parce qu'il répond à une manière de percevoir notre société.

Pour appliquer ce principe de territorialité de l'administration publique, qui devrait refléter le mieux les besoins de notre société contemporaine, nous pouvons emprunter divers chemins, autant dans des systèmes fédéraux que dans des systèmes de type unitaire. Les modes d'action peuvent être la déconcentration ou la décentralisation, ou les deux à la fois. Ces deux notions sont juridiques. Cela 
montre que le droit peut être un instrument de transformation de notre système pour aller vers la territorialité. Mais le droit ne peut définir la territorialité en elle-même, car par cet instrument (comprenant la décentralisation et la déconcentration), nous en arrivons à transformer des systèmes politiques et administratifs différents. La déconcentration doit être certes considérée au même titre que la décentralisation. Une combinaison des deux serait peut-être la solution.

La territorialité remet en cause la traditionnelle séparation entre le privé et le public, séparation qui perd encore plus d'importance si on la situe dans le contexte de la mondialisation des marchés. C'est une tentative pour trouver de nouveaux modèles de gestion de l'État. L'impartition ${ }^{5}$ doit être aussi considérée comme une forme de décentralisation.

\section{La gestion du transport en commun par les coopératives}

Dans le contexte du retour vers la territorialité, l'approche et les solutions dans le transport urbain, comme d'ailleurs dans beaucoup de domaines, se trouvent dans les structures locales et dans des véhicules institutionnels qui sont proches de la population. À ce titre, les coopératives sont un moyen de choix, puisque ce sont les personnes du milieu qui doivent se prendre en main pour répondre à leurs besoins, en l'occurrence le transport. Les coopératives s'intègrent très bien à cette nouvelle approche de la gestion du développement par le milieu local. Dans le cas de l'Amérique latine, la gestion coopérative constitue une alternative intéressante à la multiplicité des acteurs privés.

\section{Le modèle coopératif}

Dans les principes de base de l'approche coopérative, nous retrouvons plusieurs éléments de compromis qui pourraient permettre la mise en place d'un système mieux régulé, et qui toucheraient tant le cas des opérateurs que des usagers.

Ces principes sont :

- l'adhésion volontaire et ouverte à tous ;

- le pouvoir démocratique exercé par les membres ;

- la participation économique assurée pour les membres ;
- l'autonomie et l'indépendance de la coopérative, préservées dans l'optique des rapports avec des organisations tierces (y compris le gouvernement et les institutions de financement);

- l'éducation, la formation et l'information planifiées;

- la coopération entre les coopératives (des alliances sont possibles entre différentes coopératives de transport telles coopérative pour le transport des handicapés, coopérative de maintenance du matériel et coopérative d'agglomération qui viserait à assurer une coordination régionale du transport en commun);

- l'engagement envers la communauté (cet aspect est souvent moins important dans le cas d'une gestion assurée uniquement par le privé).

À l'heure actuelle, le modèle coopératif est présent dans le monde entier. Son utilité et son efficacité en font une structure d'entreprise privilégiée, comme les statistiques le montrent: il existe 750000 coopératives réparties dans 93 pays des cinq continents, et comptant près de 726 millions de membres ${ }^{6}$.

\section{La coopérative comme outil de gestion du transport en commun}

De par sa nature, la coopérative peut s'avérer un outil de gestion original et opportun dans le domaine du transport. Il est possible d'imaginer des coopératives de consommateurs (transport en commun, transport adapté, transport pour handicapés, covoiturage ${ }^{7}$, entretien de véhicules, etc.), des coopératives d'opérateurs (producteurs), qui fournissent le service, comme des coopératives de taxi, de transport en commun (autobus, train, métro), etc.

La coopérative fournit une structure organisationnelle intéressante, qui permet souvent de pallier les inconvénients des autres associations à caractère économique, en ce qu'elle a l'avantage d'être plus près des individus, et donc de leurs besoins. C'est en réalité l'une des seules institutions qui permet de conjuguer la dimension sociale et la dimension économique. En plus de fournir une structure, la coopérative nécessite une prise en charge du milieu, qui a pour conséquence de faciliter et d'améliorer les chances de répondre convenablement aux besoins ressentis par ce même milieu.

L'approche coopérative peut concilier l'intérêt des utilisateurs (coopérative d'usagers) et des opérateurs 
(coopératives de propriétaires). Elle a l'avantage d'être un compromis entre une privatisation et une étatisation. La formule coopérative peut apporter une régulation du service, parfois déficiente dans le cas de la privatisation. En même temps, elle permet d'éviter les lourdeurs d'une étatisation, qui peut être un modèle de gestion inapplicable dans ce cas spécifique, car politiquement, il occasionne un désengagement trop important du secteur privé (la survie économique de plusieurs opérateurs étant mise en péril).

\section{L'approche coopérative peut concilier l'intérêt des utilisateurs (coopérative d'usagers) et des opérateurs (coopératives de propriétaires). Elle a l'avantage d'être un compromis entre une privatisation et une étatisation.}

La modernisation de l'administration ou des services publics implique avant tout une volonté ferme des gens du milieu de susciter un changement. Dans ce cas, le nombre de propriétaires d'entreprises concernés, jumelé au nombre d'usagés, complique de beaucoup la démarche pour améliorer ce service public. Chacun doit y trouver une certaine équité, tant du côté des usagers (augmentation de la qualité du service) que des propriétaires (survie économique de ces intervenants, meilleure rationalisation en effectuant certains regroupements).

Mais l'approche coopérative n'est pas le seul moyen intéressant; d'autres le sont également, comme la décentralisation de l'administration publique, la déconcentration, les sociétés mixtes, la privatisation, etc. Dans la prochaine partie, nous établirons une comparaison sommaire de ces approches pour tenter d'en tirer certaines pistes de solution et de dégager les avantages et les inconvénients de chacun.

\section{Les autres modes de gestion possibles du transport en commun dans le contexte du retour vers la territorialité}

Dans la situation actuelle, les gouvernements supérieurs doivent imaginer, inventer et «importer» de nouvelles techniques de gestion, favorisant de nouvelles formes de partenariat.

Comme ils sont fermement déterminés à freiner l'endettement public, la participation active du secteur privé à l'effort économique et social constitue une voie intéressante.
Plusieurs solutions sont alors possibles pour établir des modes de gestion performants dans le domaine du transport en commun. Ces différents modes de gestion sont synthétisés dans le tableau 1 .

La problématique du transport en commun dans certains pays d'Amérique du Sud et d'Amérique centrale est porteuse d'enseignements en ce qui concerne la modernisation de l'appareil administratif public. En effet, dans ce cas spécifique, on vit un peu le problème inverse que l'on rencontre dans le processus de décentralisation des pouvoirs de l'État. En général, et selon la tendance du «retour vers la territorialité », que nous avons défini auparavant, pour l'ensemble des problématiques, nous devons penser en termes de décentralisation, de déconcentration et de privatisation; les solutions recherchées sont envisagées en fonction d'un désengagement de l'État dans les affaires publiques. Les modes de gestion choisis provoquent une dérégulation des règles administratives et gouvernementales. Dans le cas de la problématique du transport en commun dans ces régions du monde, nous faisons face au phénomène inverse. Les organismes de transport sont dans plusieurs cas privés et forment une constellation de petites entreprises, qui génèrent un manque de coordination et d'efficacité de ce service. Ces problèmes ont un effet direct sur le développement économique et social des pays concernés.

En Amérique latine, l'une des phases les plus importantes du processus de réalisation consisterait à créer des entreprises modernes de transport, en amorçant le processus de regroupement des opérateurs isolés et en jetant les bases institutionnelles favorables à leur éclosion. En effet, l'un des problèmes majeurs étant le trop grand nombre de transporteurs privés, plusieurs problèmes de gestion se posent, comme la rentabilité économique, le manque de coordination et l'inefficacité du service.

La structure de financement de ces entités doit être choisie avec soin. Il pourrait s'agir d'une tarification élevée pour assurer un financement par les usagers, d'une tarification sociale visant à privilégier certaines catégories d'usagers (jeunes, femmes accompagnées d'enfants, personnes âgées, etc.), d'une coopérative de transporteurs et/ou d'usagers comportant un financement de type coopératif, d'un système de subvention conditionnelle et inconditionnelle, laissant un certain contrôle à l'État, ou encore d'une participation directe de l'entreprise et du commerce au financement. Un choix éclairé devrait tenir compte des facteurs économiques, sociaux, culturels et politiques du milieu. 


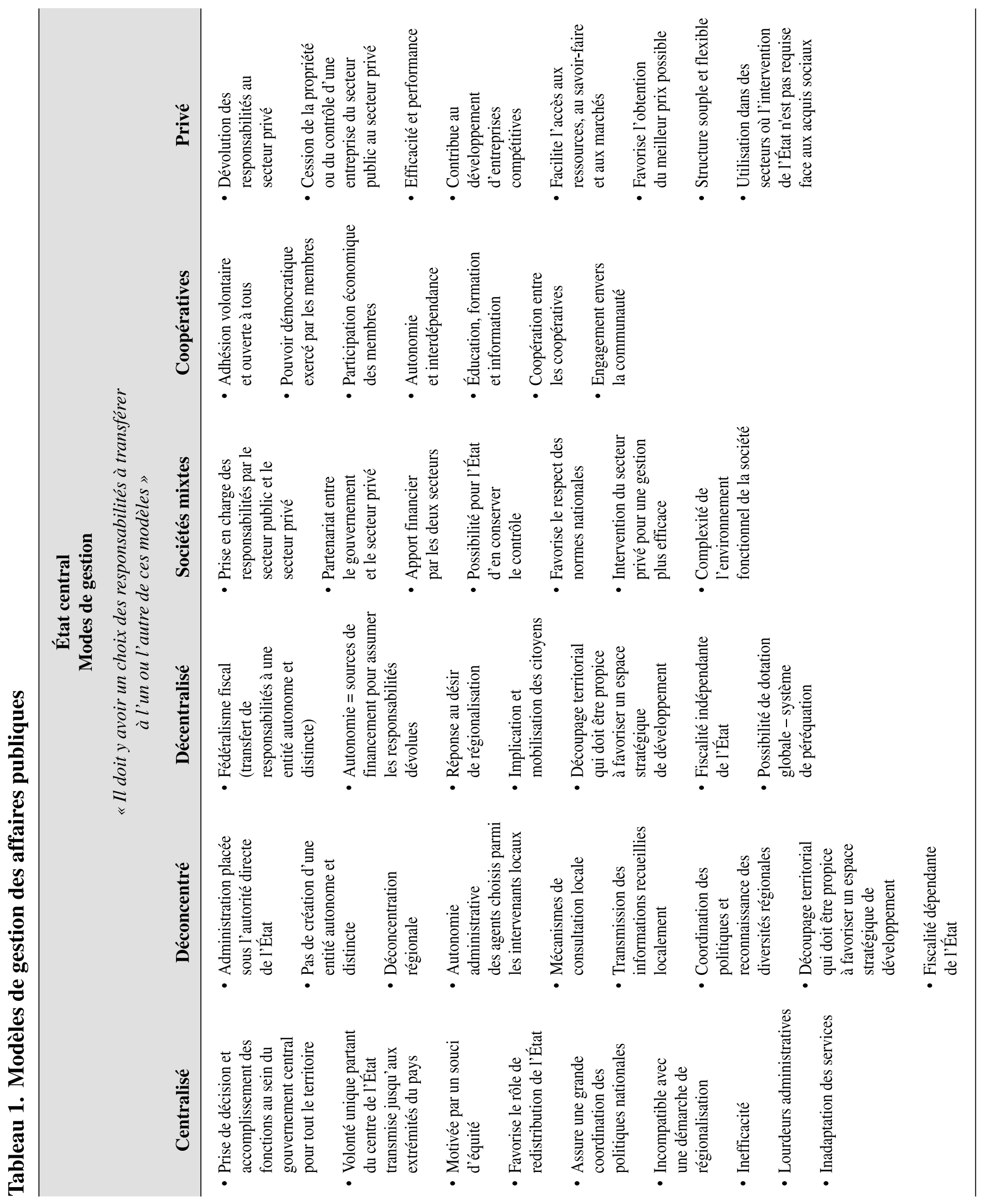


Nous pouvons décoder un effet pervers provoqué par un état de privatisation trop accentué de certains services publics, qui mène à un dysfonctionnement du système et à un manque de coordination. Dans ce cas particulier, les voies proposées iraient vers la recherche d'une plus grande implication directe de l'État (création d'une société publique de transport, étatisation) ou bien vers des solutions que l'on peut qualifier d'hybrides, en ce qu'elles s'appuient sur une implication concertée du secteur public et du secteur privé, à titre d'exemple les sociétés mixtes.

Les voies proposées iraient vers la recherche d'une plus grande implication directe de l'État (création d'une société publique de transport, étatisation) ou bien vers des solutions que I'on peut qualifier d'hybrides, en ce qu'elles s'appuient sur une implication concertée du secteur public et du secteur privé, à titre d'exemple les sociétés mixtes.

Cette dernière voie peut être aussi intéressante. C'est une institution, d'ailleurs fort bien connue en France, que l'on appelle «société d'économie mixte». Ce nouvel outil de participation s'inscrit très bien dans l'approche générale et plus particulière de la territorialité développée au début de notre exposé.

Essentiellement, la société d'économie mixte favorise, dans la mise en place d'une entreprise commune, le partenariat d'intervenants qui proviennent du secteur public et du secteur privé. À l'intérieur d'une telle association, les intervenants mettent en commun leurs différentes ressources en vue de fournir le plus souvent des services, qui seront d'intérêt public. Cette formule présente l'avantage d'alléger le fardeau financier des différents paliers de gouvernements sans pour autant alourdir le fardeau fiscal des contribuables.

Dans le cas du transport en commun, on pourrait peut-être envisager un mode de gestion comportant un partenariat entre l'État, le secteur privé conventionnel et la coopérative. Il pourrait s'agir d'une piste intéressante de recherche, qui pourrait permettre de trouver un compromis viable dans certaines situations.

Quant aux autres possibilités d'établissement d'un mode de gestion, qu'il s'agisse de l'approche centralisée, décentralisée, déconcentrée ou bien privée, nous référons le lecteur au tableau 1, qui expose les critères de base de ces modes de gestion tout en nous permettant de les comparer.

\section{Conclusion}

L'approche coopérative peut s'avérer un instrument précieux dans la recherche de modes de gestion appropriés au transport en commun en Amérique latine. Trop souvent, dans le processus de modernisation de l'administration publique, et dans la création de nouvelles politiques liées aux services publics, la tendance vise à rechercher des solutions qui sont campées strictement dans les modes de gestion publique ou bien dans les modes de gestion privée. Il s'agit là de deux extrémités en ce qui a trait aux manières de faire, les partisans de chacune des deux approches, pour des raisons sans doute valables, évoquant les avantages qui sont propres à leur approche respective.

Sans vouloir relancer le débat sur la privatisation des services publics dans le cadre de notre exposé, il est intéressant de rechercher des solutions de gestion dites intermédiaires. Dans ce contexte, l'approche coopérative, pour les raisons que nous avons décrites dans notre texte, s'inscrit certainement comme une alternative intéressante. Cette approche est souvent peu connue des gestionnaires et des experts dans le domaine des services publics, et malheureusement ignorée pour cette seule raison. Elle s'intègre bien dans l'approche plus générale du développement local et devient une façon à la fois originale et efficace de gérer une partie des activités en milieu local.

Par ailleurs, la mondialisation nous oblige à innover pour conserver un équilibre nécessaire dans nos sociétés. À cette fin, il faut favoriser l'implication de toutes les couches de nos sociétés. Cette position est valable autant pour les pays en développement que pour les pays développés.

La modernisation de l'appareil administratif, en particulier dans l'organisation de la gestion du transport en commun dans les agglomérations et dans leurs banlieues, nécessiterait la mise en place d'une structure régionale de coordination. Cette structure peut se comparer à une agence de transport métropolitaine.

Cette structure régionale de transport fonctionnerait sur la base d'une approche de gestion et de financement coopératif. Nous n'avons pu trouver de modèle correspondant de près ou de loin à cette approche de gestion. Cependant, quand on connaît les problèmes de gestion et 
de financement rencontrés par les structures régionales de transport tant en Amérique du Sud et en Amérique centrale qu'en Amérique du Nord, on peut s'interroger sérieusement sur la possibilité d'implanter cette nouvelle approche. Il s'agirait certainement d'une piste de recherche des plus intéressante à développer et qui pourrait apporter des éléments de modernisation des plus profitables.

Le développement des pays d'Amérique latine passe obligatoirement par une amélioration du service public de transport en commun. C'est aussi une condition de base pour permettre l'implication de toutes les couches de la société dans ce processus. Ainsi, dans la recherche de solutions à cette problématique du transport en commun, la gestion coopérative nous apparaît à la fois comme une solution et comme une manière additionnelle de permettre l'intégration de l'ensemble de la population dans le processus de développement.

\section{Notes et références}

1 Jacques Gagnon est professeur au département de management de la Faculté d'administration de l'Université de Sherbrooke, titulaire de la Chaire J.W. McConnell en gestion du développement local, directeur scientifique du Groupe de recherche en administration publique et membre du Centre d'excellence en gestion du développement local. Peggy Bachman est doctorante au D.B.A. de l'Université de Sherbrooke et directrice scientifique adjointe du Groupe de recherche en administration publique.

2 Les grandes politiques sectorielles n'ont pu régler des problèmes de fond de notre société (ex. : chômage et immigration) et l'on doit maintenant penser plus globalement mais à un niveau territorial.

3 Cette mise de côté comprend peut-être le retour aux subventions conditionnelles et inconditionnelles.

4 GLEIZAL, J. J., J. GATTI DOMENACH et C. JOURNÈS (1993). La police. Le cas des démocraties occidentales, Paris, Presses universitaires de France, Thémis, p. 345-346.

5 L'impartition peut être envisagée sous des formes dites moins agressives qui réservent un pouvoir plus important aux communautés telles le modèle coopératif et les sociétés mixtes.

6 Alliance coopérative internationale, ICA Statistics-1998, disponible au www.coop.org/statistics.html

7 GRENIER, Louis (1999). The Calgary Alternative Transportation Co-operative, Mémoire de maîtrise, Université de Sherbrooke (IRECUS), p. 4-5. 